\title{
Handover process: how to improve quality and safety through an ergonomic solution
}

\author{
Giulio Toccafondi ${ }^{\mathrm{a}}$, Sara Albolino ${ }^{\mathrm{a}}$, Tommaso Bellandi ${ }^{\mathrm{a}}$ and Francesco Venneri ${ }^{\mathrm{b}}$ \\ ${ }^{a}$ Patient Safety and Clinical Risk Management Tuscany Region, Taddeo Alderotti 26N , 50129 Florence, Italy \\ ${ }^{\mathrm{b}}$ Clinical Risk Management Unit, ASF10, Santa Maria Nuova 1, Florence, Italy
}

\begin{abstract}
Patient safety practices for enhancing the quality and safety of handover are context sensitive interventions. In this article we explore the use of cultural probes as a qualitative technique with a twofold objective: eliciting implicit activity pattern and tools that may constitute resources for the design of effective handover solutions and prompting health care practitioners' participation and involvement.
\end{abstract}

Keywords: handover, human factors, design, participation

\section{Introduction}

Communication modalities of medical information are crucial in complex health care systems. The handover process during shift changes and transitions of care is strategic for quality and safety. The Joint Commission reports the lack of an effective communication as the root cause analysis for the majority of sentinel events happened from 1992 to 2006 in the United States [1]. After that, many studies and projects [2-4,11] have shown how an effective communication during the exchange of medial information is a key element to avoid adverse events and improve the efficacy of the multisciplinary process of care. Handover in healthcare is "the transfers of responsibility and accountability for a patient from one care giver to another" [5]. Research outlined that the quality of handover process is not uniquely underpinned by effective information transfer. Handover is a complex multimodal communication process [6]. A blend of components contributes to the quality of medical information transfer: standardization of content, type of media, quality and safety culture, and organizational climate. The Handover EU project [3] showed how transitions of care are a context sensitive practice dependent on organizational culture, beliefs, and human factors. In many health care setting there are not official guidelines and codified best practices defining the communication modalities for medical information and transfer of role among care professionals. The handover process is carried out through implicit and not codified activity patterns. The lack of a shared model may lead to a potential risks when variations occur e.g. change of medical teams, transitions from high acuity to low acuity. The Centre for Clinical Risk Management and Patient Safety of the Tuscany Region (GRC) is promoting a pilot study which involves 6 local healthcare agencies, 20 units and more than 200 professionals. The main objective of the pilot study is to improve patient safety in handover process through the definition of an ergonomic solution centered on effective communication. The project will focus on the analysis of the handover processes between high acuity (ER and the Intensive care Unit) and low acuity care (wards of surgical units) indicated as at high risk in the literature [9]. The pilot study aims at acquiring data regarding the current handover processes in order to elicit the technical, nontechnical and contextual aspects. The pilot study will analyze from a human factors and communicative perspective the tools and approach used to mediate the handoffs of medical information between homogeneous (during shift changes) and inhomogeneous groups of clinicians (during transitions of care). This paper presents the rationale and the qualitative tool - cultural probe [8] - used for triggering the analysis of in-situ handover processes in the selected health care settings. 


\section{Methods: handover as an interactive process}

Given the context sensitiveness of handover, improvements needs to be pursued not only through the refinement of discharge instructions but also by means of participative endeavors. In this pilot study the collaboration with healthcare practitioners is considered fundamental for the definition of the project mission. Thus the project mission will be formulated and defined once the activity analysis is closed and the design brief for implementing local solutions to handover issues is shared among the local workgroups.

The study departed from the hypothesis that handover practices in order to have a systemic impact need to couple in a single unit of analysis both the 'sender' and the 'recipient'. The study objects are the internal handover process, (i.e. shift changes) and the handover between different health care units (i.e. transitions of care). The handover between the health care units needs to be considered and improved as a single interactive communication process. For this reason the workgroup will observe through the cultural probes how the handover occurs during shifts changes and transitions of care between different medical staff.

\subsection{Project Phases}

In order to acquire the information conveying the needs and opportunities to inform the design phase of local solutions the project is structured in the following phases:

Phase 1. State of the art. This first stage is aimed at the literature review analysis and sharing with pilot study group of the state of the art of experiences. Phase 2. Definition of the group for the pilot. In each local healthcare agency involved the group is coordinated by the local clinical risk manager and for each unit a physician is the leader for the project. According to the user centered approach, the design of the solution is co-operative, every clinician, interested in the process, participates in the process of analyzing the handover process and defining new solutions.

Phase 3. Activity analysis of the handover process through the use of the cultural probe. The objective of this phase is to map the handovers process by taking into consideration the tools and the working patterns used to carry out the transfer of medical information. In particular the sub objectives of this phase are
- Definition of a preliminary general model of handover based on interviews and ethnographic observations through cultural probe

- The collection of tools actually used for supporting handover

- The kick-off of the prototyping of the cultural probe

Phase 4. Definition of local ergonomic solution and definition of indicators for the evaluation. According to the main results of the analysis, this phase is dedicated to the design, with the participation of users, of an ergonomic solution aimed at improving the quality and safety of the handover process studied. Together with the process redesign it is fundamental to define some quality and safety indicators to monitor the impact of the new practices.

\subsection{Observation through Cultural Probes}

The practitioners with the support of the Risk Management staff perform the observation supported by the cultural probe. The cultural probe mediates the observation and it aims at triggering the practitioners' reflection on the handover process. The observer and the observed are equally involved in the process of understanding what is happening during the handover process and what types of tools and work patterns are mediating the activity. The cultural probes are two different structured grids (see fig, 1 and 2), one is used for the analysis of handovers practices during the shift changes, the other one is used for the analysis of the handover practices during transition of care. The collected data are the input for the organization of Focus Group based on the FMEA technique aimed at detecting and codifying the main criticalities associated to the analyzed handover practices. The observations are complemented with the collection of formal and informal documents (e.g. personal notes informal checklist) and of any kind of artifact that supports or is related to the handover processes.

\subsection{Shift Change and Transitions of Care Handover}

The shifts changes handover is focused on two patients. For each patient are observed all the exchanges of medical information and transfer of responsibility occurring during a full day (24 hours). For the Emergency Room the selected patients have to be classified with green and yellow tag according 
to the triage code. For the Intensive Care Unit the two selected patients need to be evaluated according to Glasgow scale score. The observation is autonomously managed by each care unit, and has to be done by an operator that is not involved in the handover process. For the handover during transition of care, the observation is focused on ten patients. It is strongly encouraged the inclusion of the patients previously considered for the shifts changes handover. The observation involves both the unit that discharges (sender) the patient and the unit that admits the patient (recipient). For the Emergency Room five patients have to be classified with green tag according to the triage code while the other five have to be classified with yellow tag. For the Intensive care Unit the choice of the patients is discretional but the Glasgow scale score has to be indicated. Also for this observation, the operator who observes must not be involved in the handover process and he/she is the same for both observed units. Before the elaboration of the structured observations, the researchers have conducted a research scouting phase on the field where they visited the involved units and they conducted some unstructured interview and some interactive observations aimed at better understanding the context and shaping the cultural probes.

\section{Prototyping the Cultural Probe}

The main objective of the cultural probe is to analyze the handover process in order to understand how it is actually performed, to identify critical aspects and to eventually enhance the activity pattern mediating the whole process. Observations of in-situ handover are performed in order to outline the components of the current handovers during shift changes and during transitions of care. The probe's components were graphically represented in paper mock-up. The prototype was validated with the health care practitioners in iterative cycles of design. The cultural probe is aimed at involving the health care practitioners in collecting data on handover process from an user centered design approach and at exploring the following issues:

- Is there a logical structure underpinning handover?

- Is there a correlation between the shift change (internal handover) process and the transitions of care process (external handover)?

- Which actors are involved in the handover process?
- Which tools mediate the communication of medical information?

- What modalities are used to communicate medical information?

- What information is directly connected with the care actions?

- How should information been transmitted in order not to become redundant?

- Does redundancy generate safety or it is likely that the most important information get nested in too much textual information?

- Is any piece of relevant information lost during the handover?

- Is the retrieval of information in the medical documentation straightforward after the transitions?

- What kind of communication exchanges prepare the transitions of the patients?

- What is the content of such communications?

The documentation may not reflect actual discharge process and the mere transfer of information does not ensure the quality of handover process.

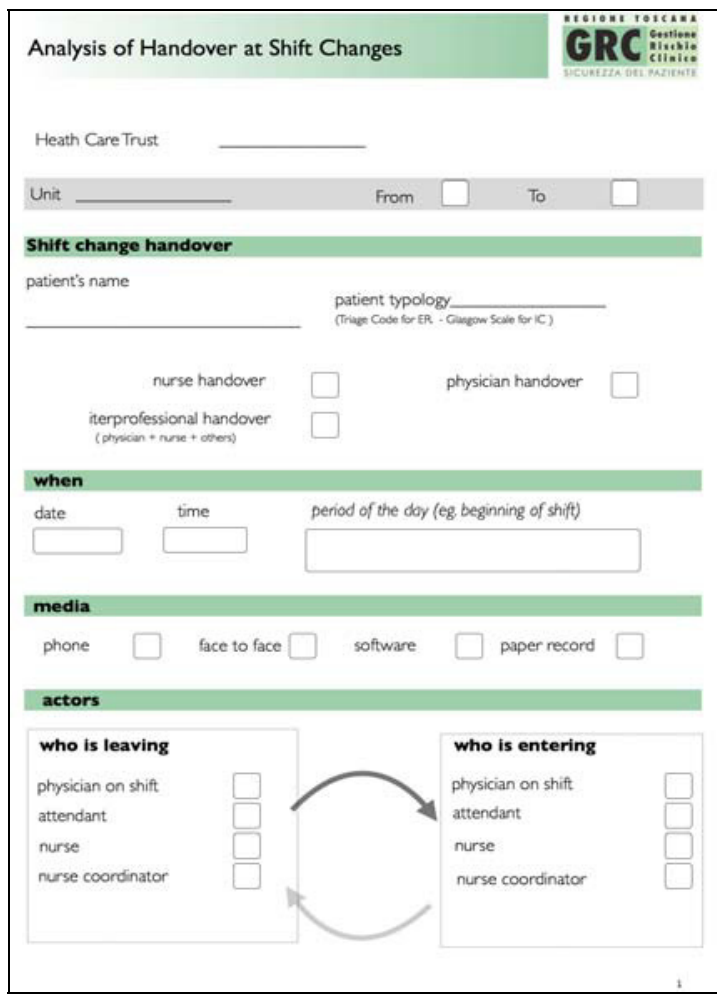

Figure 1 -cultural probe, section for shift change handover 
The efficacy of the communication at transitions of care may be hindered by issues related to "shared understanding" [10] e.g. different ways to read the documentation or a different disciplinary stance. The continuity of information among different teams may be limited by omission of relevant information, difficulties in retrieving the medical information in the textual documentation. Moreover, the probes may capture best practices in the communication of medical information transferable to other contexts. The probe is graphically structured in subsequent phases and it is designed to be filled during the handover process. The probes trace the typology of actors involved, the kind of handover performed, the numbers of contacts preparing the care transitions, the tools mediating it, the types of information communicated, and the perceived relevance of the information transmitted (see fig.2).

\subsection{Identifying Actors, Tools and Criticalities}

The observation supported by the cultural probe is meant to construct a base for a shared reflection on the handover of medical information between the sender and recipient unit. Secondly, the probe is meant to prompt discussion and debate on the potential omissions and variations occurring when patients are transferred from high acuity care to low acuity care. During transitions of care the documentation is usually reorganized in order to provide a synthetized short medical report. It may constitute a potential criticality the fact that while the documentation is transferred to the other unit, it is not always transferred the way in which the clinical documentation should be read and linked to the synthesis. The outcomes of the cultural probes and the experiences of the operators personally involved in the analysis will provide the base for Focus Group sessions. The aim of the sessions is to bring together the two different care units interacting during the handover process and develop a FMEA analysis of the work patterns and tools mediating handover. Through a participative approach the knowledge produced during the focus group will be used to produce a flow diagram of the communication flow. The objective of this phase is the construction of a flow diagrams which explicitly represents the handover of the two units. Thus, the objective is the construction of a system overview in which the different kind of handovers (shift changes transition of care) are jointly represented and mutually considered as part of a single system.

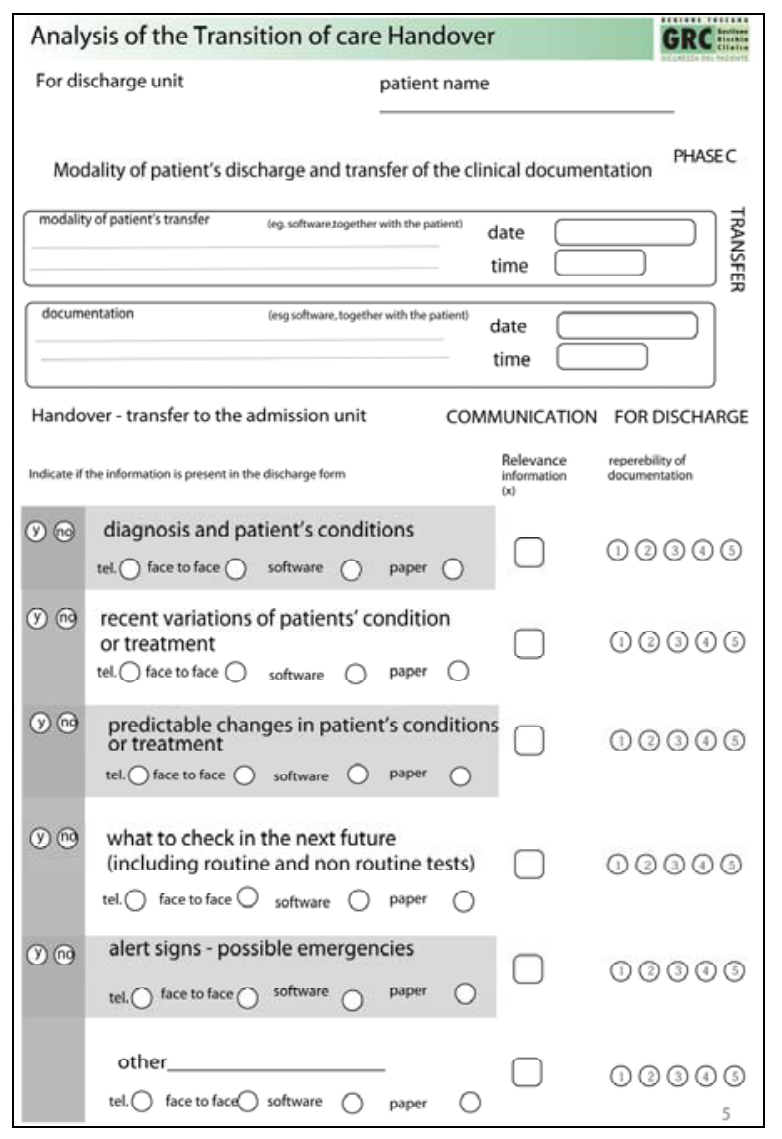

Figure 2 - cultural probe for transition of care handover

\section{Critical Evaluation}

The pilot study outlined in this article is designed as an open platform for the involvement of health care practitioners. As previous endeavors have shown [2] the participation and involvement of health care practitioners is a requirement for the definition of solutions regarding handover. The relevance of contextual factors in the dissemination and implementation of patient safety practice is widely acknowledged as an element that research activity should explicitly take into consideration [7]. As the health care system is becoming more complex, due to centralization of services, the deployment of practices reducing the variability throughout the process of medical information communication is needed. The involvement of healthcare practitioners in the activity of system redesign is therefore a viable strategy that needs, in order to be applied, a flexible and open project format that consider the activity analysis of the context of interventions a necessary premises to the design activity. 


\section{Conclusion}

The design of a pilot study based on the health care practitioners involvement stems from the need of the implementation of context sensitive patient safety practices. The application of the cultural probe technique within a qualitative approach provides new opportunities not only for supporting a participative approach but also for the collection of experiential data connected to highly context dependent practice such as the communication of medical information in high risk health care settings.

\section{References}

[1] WHO patient Safety Solution 3, WHO Press Geneva 2007

[2] Australian Commission on Safety and Quality in Health Care (2010). The OSSIE Guide to Clinical Handover Improvement. Sydney, ACSQHC.

[3] NHS, Safe handover: safe patients -2004

[4] www.handover.eu

[5] Cummings, E., Showell, C., Roehrer, E., Churchill, B., Turner, B., Yee, K.C., Wong, M.C.,Turner, P. (2010) Discharge, Referral and Admission: A Structured Evidence-based Literature Review, eHealth Services Research Group, University of Tasmania, Australia.
[6] Manser T, Foster S, Gisin S, Jaeckel D, Ummenhofer W. Assessing the quality of patient handoffs at care transitions. Qual Saf Health Care. 2010 Dec;19(6).

[7] Shekelle PG, Pronovost PJ, Wachter RM, Taylor SL, Dy S, Foy R, Hempel S, McDonald, K, Ovretveit J, Rubenstein L, and the PSP Technical Expert Panel. Assessing the Evidence for Context-Sensitive Effectiveness and Safety of Patient Safety Practices: Developing Criteria. Contract Final Report. AHRQ Publication No. 11-0006-EF, December 2010. Prepared under Contract No. HHSA-290-2009-10001C. Agency for Healthcare Research and Quality, Rockville, MD. http://www.ahrq.gov/qual/contextsensitive/

[8] Gaver, B., Dunne, T., Pacenti, E. Design: Cultural Probes, Interaction volume 6 issue $1, \mathrm{ACM}$ press 1999

[9] Implementation Toolkit - Standard Key Principle for Clinical Handover, NSW - Australia, Department of Health, 2009

[10]Tomasello, M., Carpenter, M., Call, J.,Behne, T., and Moll, H., Understanding and sharing intentions: The origins of cultural cognition, Behavioral and Brain Science (2005) 28, 675-735

[11] Bahbra et al An experimental comparison of several handover methods in Annals of the Royal College of Surgeon of England 2007; 89: 298-300 